\title{
Resource Allocation for MU-MIMO Non-Orthogonal Multiple Access (NOMA) System with Interference Alignment
}

DOI:

10.1109/ICC.2017.7996956

\section{Document Version}

Accepted author manuscript

Link to publication record in Manchester Research Explorer

\section{Citation for published version (APA):}

Al-Abbasi, Z., So, K. C., \& Tang, J. (2017). Resource Allocation for MU-MIMO Non-Orthogonal Multiple Access (NOMA) System with Interference Alignment. In IEEE International Conference on Communications IEEE Computer Society . https://doi.org/10.1109/ICC.2017.7996956

\section{Published in:}

IEEE International Conference on Communications

\section{Citing this paper}

Please note that where the full-text provided on Manchester Research Explorer is the Author Accepted Manuscript or Proof version this may differ from the final Published version. If citing, it is advised that you check and use the publisher's definitive version.

\section{General rights}

Copyright and moral rights for the publications made accessible in the Research Explorer are retained by the authors and/or other copyright owners and it is a condition of accessing publications that users recognise and abide by the legal requirements associated with these rights.

\section{Takedown policy}

If you believe that this document breaches copyright please refer to the University of Manchester's Takedown Procedures [http://man.ac.uk/04Y6Bo] or contact uml.scholarlycommunications@manchester.ac.uk providing relevant details, so we can investigate your claim.

\section{OPEN ACCESS}




\title{
Resource Allocation for MU-MIMO Non-Orthogonal Multiple Access (NOMA) System with Interference Alignment
}

\author{
Ziad Qais Al-Abbasi ${ }^{\dagger}$, Daniel K. C. So ${ }^{\dagger}$, and Jie Tang ${ }^{\ddagger}$ \\ School of Electrical and Electronic Engineering ${ }^{\dagger}$ \\ The University of Manchester ${ }^{\dagger}$, United Kingdom, South China University of Technology ${ }^{\ddagger}$, China \\ Email: ziad.al-abbasi@manchester.ac.uk; d.so@manchester.ac.uk; eejtang@scut.edu.cn
}

\begin{abstract}
Non-orthogonal multiple access (NOMA) has attracted a lot of attention recently due to its superior spectral efficiency and could play a vital role in improving the capacity of future networks. This paper considers resource allocation for a downlink, multi-user (MU) MIMO-NOMA system that aims at maximizing the sum rate with interference alignment (IA) technique. Using singular decomposition value (SVD) based IA, we propose IA based NOMA system in which a number of users are grouped together while the others are aligned to the nul space as interference. The targeted group of users employ NOMA with a low complexity hierarchical power allocation scheme for sum rate maximization. In addition, an optimization problem is formulated to maximize the sum rate under the total power and proportional fairness constraints. A low complexity sub-optimal solution for two-user scenario is obtained and then extended to the multi-user case by a hierarchical pairing scheme. Another approach is proposed to allocate the transmission power of each user using an iterative subgradient method. Simulation results show that the proposed schemes provide better performance than an existing scheme and perform close to the optimal one. In addition, the simulation scenario considers the case where two users share the data streams while performing IA as compared to the case where all users are sharing it without IA. Simulation results verify that applying IA with NOMA could improve the achievable sum rate and offers simplicity in terms of successive interference cancellation (SIC) application.
\end{abstract}

Index Terms-Multiple input multiple output (MIMO) system, Non-orthogonal multiple access (NOMA), resource allocation, sum rate maximization, interference alignment.

\section{INTRODUCTION}

In orthogonal multiple access systems, the users and the base station (BS) communicates through an orthogonal channel via a dedicated radio resource [1], [2]. However, this approach not spectral efficient as the radio resources segmented and are not being used to its full. To increase system capacity and reduce latency in the next generation of mobile networks, it is better to allow the BS-users communications to be simultaneously through all the available resource in a non-orthogonal manner. This is made possible by using nonorthogonal multiple access (NOMA), where recent articles demonstrated its potential as one of the key air interface technologies to outperform orthogonal multiple access (OMA) and achieves the capacity region of a downlink AWGN channel [3]-[5]. The idea of NOMA is that it allows the users to share resources in time and frequency by multiplexing the users in the power domain. At the receiver side, users' detection is realized by applying successive interference cancellation (SIC).

Recent works suggest that further enhancement of NOMA performance is possible by combining it with multiple input multiple output (MIMO) techniques since the use of MIMO provides additional degrees of freedom [4], [6]-[10]. The authors in [6] proposed user-clustering algorithm for a multiuser MIMO-NOMA system. With two users per cluster, the power is allocated based on fractional transmit power allocation (FTPA) scheme; the authors optimized the precoding matrix with an objective to maximize the achievable sum rate. The problem is solved based on the Majorization Minimization (MM) approach. However, it is unclear how the clustering will be for odd number of users, or when the number of users is more than double the number of the antennas. A transmission framework for the downlink and uplink of MIMO-NOMA system is proposed in [4] using signals alignment. The authors also addressed fixed and cognitive power allocation to evaluate NOMA system performance. In addition, [7] investigated the performance of MIMO-NOMA and MIMO based orthogonal multiple access (OMA) and demonstrated that the former offers better capacity than the latter. However, in both [4], [7], each base station subspace is used to serve a cluster of two users only.

In this paper, we propose applying interference alignment (IA) to MIMO-NOMA system, in which two or more users are grouped together while the others are aligned to the null space as interference. The targeted group of users use NOMA with a low complexity hierarchical power allocation scheme for sum rate maximization. In addition, we propose two power allocation schemes to allocate the power to the targeted users. A sum rate maximization problem is formulated subject to the total power and the minimum rate constraints. After that, a low complexity sub-optimal solution for twouser scenario is obtained and then extended to the multiuser case by a hierarchical pairing scheme. Another approach is also proposed to allocate the transmission power of each user using an iterative subgradient method. Simulation results demonstrate the effectiveness of IA in enhancing NOMA performance and simplifying the SIC application. The results 
also verify the effectiveness of the proposed power allocation schemes as compared to the optimal one and the FTPA-NOMA from [6]. The rest of this paper is organized as follows. Section II addresses the system model of the downlink NOMA system. It also presents the formulated optimization problem, the proposed suboptimal solution, and the subgradient based power allocation scheme. The simulation scenario and results are discussed in Section III. Finally, Section IV concludes the paper.

\section{SySTEM MOdEL}

A downlink MU-MIMO system consisting of one BS equipped with $M$ antennas and $K$ users each equipped with $N$ antennas is considered. Using power domain multiplexing, the transmitted signal from the BS to the $k$-th user will share the same time-frequency resource and it is given by

$$
\mathbf{x}^{(k)}=\mathbf{V}^{(k)} \tilde{\mathbf{s}}
$$

where $\mathbf{V}^{(k)} \in \mathbb{C}^{M \times S}$ stands for the precoding matrix used by the BS, $\tilde{\mathbf{s}} \in \mathbb{C}^{S \times 1}$ is the information bearing vector that contains $S$ data streams, following NOMA concept each these stream will be shared by $K$-users, hence $\tilde{\mathrm{s}}$ is given by

$\widetilde{\mathbf{s}}=\left[\begin{array}{c}\hat{\mathbf{s}}_{1} \\ \hat{\mathbf{s}}_{2} \\ \cdot \\ \cdot \\ \hat{\mathbf{s}}_{l} \\ \vdots \\ \hat{\mathbf{s}}_{S}\end{array}\right]=\left[\begin{array}{c}P_{1}^{(1)} \mathbf{s}_{1}^{(1)}+P_{1}^{(2)} \mathbf{s}_{1}^{(2)} \ldots+P_{1}^{(k)} \mathbf{s}_{1}^{(k)} \ldots+P_{1}^{(K)} \mathbf{s}_{1}^{(K)} \\ P_{2}^{(1)} \mathbf{s}_{2}^{(1)}+P_{2}^{(2)} \mathbf{s}_{2}^{(2)} \ldots+P_{2}^{(k)} \mathbf{s}_{2}^{(k)} \ldots+P_{2}^{(K)} \mathbf{s}_{2}^{(K)} \\ \vdots \\ \cdot \\ P_{l}^{(1)} \mathbf{s}_{l}^{(1)}+P_{l}^{(2)} \mathbf{s}_{l}^{(2)} \ldots+P_{l}^{(k)} \mathbf{s}_{l}^{(k)} \ldots+P_{l}^{(K)} \mathbf{s}_{l}^{(K)} \\ \vdots \\ P_{S}^{(1)} \mathbf{s}_{S}^{(1)}+P_{S}^{(2)} \mathbf{s}_{S}^{(2)} \ldots+P_{S}^{(k)} \mathbf{s}_{S}^{(k)} \ldots+P_{S}^{(K)} \mathbf{s}_{S}^{(K)}\end{array}\right]$ where $P_{l}^{(k)}$ refers to the power allocated to the $k$-th user over the $l$-th data stream. In addition, $\mathbf{s}_{l}^{(k)}$ represents the data signal intended to the $k$-th user within the $l$-th data stream. On the other hand, the received signal by the $k$-th user can be expressed as

$$
y^{(k)}=H^{(k)} \mathbf{V}^{(k)} \hat{\mathbf{s}}_{k}+\sum_{j=1, j \neq k}^{K} H^{(k)} \mathbf{V}^{(j)} \hat{\mathbf{s}}_{j}+\mathbf{n}^{(k)} .
$$

where $H^{(k)} \in \mathbb{C}^{N \times M}$ denotes the small scale fading effect between each user and the BS and $\mathbf{n}^{(k)} \in \mathbb{C}^{N \times 1}$ denotes the normalized additive white Gaussian noise observed by user $k$. By applying the receiver beamforming matrix $\mathbf{U}^{(k)} \in \mathbb{C}^{N \times S}$, the detected signal can be modeled as

$$
\begin{aligned}
\widetilde{y}^{(k)} & =\mathbf{U}^{*(k)} y^{(k)} \\
& =\mathbf{U}^{*(k)}\left(H^{(k)} \mathbf{V}^{(k)} \hat{\mathbf{s}}_{k}+\sum_{j=1, j \neq k}^{K} H^{(k)} \mathbf{V}^{(j)} \hat{\mathbf{s}}_{j}\right)+\widetilde{\mathbf{n}}^{(k)}
\end{aligned}
$$

where ${ }^{*}$ stands for matrix Hermitian operator.

\section{A. SVD based IA}

The IA idea is to align the unwanted received signals into an interference-subspace and reducing its projection within the desired signal subspace, which is where the wanted signal directed towards [11], [12]. For example, for the $k$-th user, this is achieved by determining the precoding matrix $\mathbf{V}^{(k)}=$ $\left[\mathbf{v}_{1}^{(k)}, \mathbf{v}_{2}^{(k)}, \cdots \mathbf{v}_{l}^{(k)}, \cdots \mathbf{v s}^{(k)}\right] \in \mathbb{C}^{M \times S}$ and the post processing matrix $\mathbf{U}^{(k)}=\left[\mathbf{u}_{1}^{(k)}, \mathbf{u}_{2}^{(k)}, \cdots \mathbf{u}_{l}^{(k)}, \cdots \mathbf{u}_{S}^{(k)}\right] \in \mathbb{C}^{N \times S}$ that satisfies the following conditions

$$
\begin{gathered}
\operatorname{rank}\left(\mathbf{U}^{(k) *} \mathbf{H}^{(k)} \mathbf{V}^{(k)}\right)=S \\
\mathbf{U}^{(k) *} \mathbf{H}^{(k)} \mathbf{V}^{(j)}=0 \quad \forall j \neq k
\end{gathered}
$$

where $S \leq \min (M, N)$ which represents the number of data streams. Consider the scenario where only two users are multiplexed per each data stream, IA will be applied to align the effect of all other pairs by considering them as interference and aligning their effects into the interference subspace. This will leave each user only with the effect of its partner which means one user (the stronger user) only will apply SIC and the other user (the weaker one) will consider its partner as noise. In this paper, SVD based IA is applied as proposed in [12]. Accordingly, the equivalent (effective) channel is obtained by

$$
\tilde{\mathbf{H}}=\mathbf{U}^{(k) *} \mathbf{H}^{(k)} \mathbf{V}^{(k)} .
$$

Once the equivalent channel is identified, it can be used to design the transmit and the receive precoding matrices, where in this paper, both SVD and dirty paper coding (DPC) are used for comparison. In case of SVD, the effective channel can be decomposed as

$$
\tilde{\mathbf{H}}=\tilde{\mathbf{U}}^{(k)} \tilde{\Sigma}^{(k)} \tilde{\mathbf{V}}^{(k)}
$$

where $\tilde{\mathbf{U}}^{(k)} \in \mathbb{C}^{S \times S}$ act as a unitary matrix that contains the left singular vectors, $\tilde{\Sigma}^{(k)} \in \mathbb{C}^{S \times S}$ is a diagonal matrix with its entries represent the singular values of the effective channel, $\tilde{\mathbf{V}}^{(k)} \in \mathbb{C}^{S \times S}$ is a unitary matrix that contains the right singular vectors.

\section{B. Downlink Power Allocation}

According to NOMA principles the achievable sum rate by $K$-users is given by

$$
\begin{aligned}
& R_{s}=B_{s} \sum_{l=1}^{S} \log _{2}\left(1+\frac{P_{l}^{(K)} G_{l}^{(K)}}{B_{s} N_{0}}\right)+ \\
& B_{s} \sum_{k=1}^{K-1} \sum_{l=1}^{S} \log _{2}\left(1+\frac{P_{l}^{(k)} G_{l}^{(k)}}{B_{s} N_{0}+\sum_{j=k+1}^{K} P_{l}^{(j)} G_{l}^{(k)}}\right)
\end{aligned}
$$

where $B_{s}$ and $N_{0}$ represent the bandwidth of each resource block and the noise power spectral density respectively. $G_{l}^{(k)}$ is the effective channels of the $k$-th user and is given by

$$
G^{(k)}=\sqrt{\frac{\xi_{k}}{P L_{k}}}\left|\tilde{\mathbf{U}}^{(k)} \mathbf{H}^{(k)} \tilde{\mathbf{V}}^{(k)}\right|^{2}
$$

where $\xi$ stands for the Log-normal shadowing, $P L_{k}$ accounts for the path loss that varies with the distance $d_{k}$, which stands for the distance between the $k$-th user and the serving base station. $\tilde{\mathbf{U}}^{(k)}$ and $\tilde{\mathbf{V}}^{(k)}$ to be obtained by applying IA as explained in the previous subsection. Since $\tilde{\mathbf{U}}^{(k)}$ and $\tilde{\mathbf{V}}^{(k)}$ affect the value of $G_{l}^{(k)}$, it means that the applied IA technique affects the power allocated to each user. Using (9) to derive a 
closed form solution is very complex and as a result, we will consider a two-user scenario to derive a simpler solution. The solution is then generalized to the case with a larger number of users.

\section{Problem Formulation: Two-users based allocation}

For a two user case, the achievable rate by the user with poor channel conditions (weakest user) is given by

$$
R^{(L)}=B_{s} \sum_{l=1}^{S} \log _{2}\left(1+\gamma_{l}^{(L)}\right)
$$

while that achieved by the user with the better channel conditions (strong user) is given by

$$
R^{(H)}=B_{s} \sum_{l=1}^{S} \log _{2}\left(1+\gamma_{l}^{(H)}\right) \text {. }
$$

where

$$
\begin{gathered}
\gamma_{l}^{(H)}=\frac{P_{l}^{(H)} G_{l}^{(H)}}{B_{s} N_{0}} \\
\gamma_{l}^{(L)}=\frac{P_{l}^{(L)} G_{l}^{(L)}}{B_{s} N_{0}+P_{l}^{(H)} G_{l}^{(L)}}
\end{gathered}
$$

represent the received SINR of the stronger and weaker users in the $l$-th data stream respectively. The sum rate maximization problem is formulated as

$$
\begin{array}{ll}
\text { maximize } & R_{s} \\
\text { Subject to } & \sum_{l=1}^{S}\left(P_{l}^{(L)}+P_{l}^{(H)}\right) \leq P_{t} \\
& P_{l}^{(L)}, P_{l}^{(H)} \geq 0, \forall l, k \\
& R^{(j)}: R^{(i)} \geq \Phi_{\text {min }}^{(j)}: \Phi_{\text {min }}^{(i)} \\
& \text { where }\left(j \in\left\{1,2, \ldots, \frac{K}{2}\right\},\right. \\
& \left.i \in\left\{\frac{K}{2}+1, \frac{K}{2}+2, \ldots, K\right\}\right)
\end{array}
$$

where constraints (16) and (17) are to guarantee a positive allocated power and limited by the maximum allowable transmission power $P_{t}$. In addition, the proportional fairness constraint (18) is to control the achievable throughput by all users, where $\Phi_{\text {min }}^{(j)}$ and $\Phi_{\text {min }}^{(i)}$ are the minimum rate requirements for the user with the best channel conditions and the worst channel conditions, respectively. Using Lagrange Dual Decomposition (LDD) approach [13], the solution of (15)-(19) can be summarized as follows

$$
\begin{gathered}
F=-\mu\left(\frac{B_{s} \sum_{l=1}^{S} \log _{2}\left(1+\gamma_{l}^{(H)}\right)}{\Phi_{\min }^{(H)}}-\frac{B_{s} \sum_{l=1}^{S} \log _{2}\left(1+\gamma_{l}^{(L)}\right)}{\Phi_{\text {min }}^{(L)}}\right)+ \\
B_{s} \sum_{l=1}^{S} \log _{2}\left(1+\gamma_{l}^{(H)}\right)+B_{s} \sum_{l=1}^{S} \log _{2}\left(1+\gamma_{l}^{(L)}\right)- \\
\psi\left(P_{l}^{(L)}+P_{l}^{(H)}-P_{t}\right)
\end{gathered}
$$

where $\mu$ and $\psi$ represent the Lagrange multipliers. Differentiating against $P_{l}^{(H)}, P_{l}^{(L)}, \mu$ and $\psi$ respectively, we obtain

$$
\frac{d F}{d P_{l}^{(H)}}=\frac{G_{l}^{(H)}}{N_{0}\left(1+\gamma_{l}^{(H)}\right)}-\frac{B_{s} P_{l}^{(L)}\left(G_{l}^{(L)}\right)^{2}}{\left(B_{s} N_{0}+P_{l}^{(H)} G_{l}^{(L)}\right)^{2}\left(1+\gamma_{l}^{(L)}\right)}-
$$

$$
\begin{gathered}
\psi-\mu\left(\frac{G_{l}^{(H)}}{N_{0} \Phi_{\min }^{(H)}\left(1+\gamma_{l}^{(H)}\right)}+\right. \\
\left.\frac{B_{s} P_{l}^{(L)}\left(G_{l}^{(L)}\right)^{2}}{\Phi_{\min }^{(L)}\left(B_{s} N_{0}+P_{l}^{(H)} G_{l}^{(L)}\right)^{2}\left(1+\gamma_{l}^{(L)}\right)}\right) \\
\frac{d F}{d P_{l}^{(L)}}=\frac{\mu B_{s} G_{l}^{(L)}}{\Phi_{\min }^{(L)}\left(B_{s} N_{0}+P_{l}^{(H)} G_{l}^{(L)}\right)\left(1+\gamma_{l}^{(L)}\right)}+ \\
\frac{d F}{d \mu}=\frac{B_{s}}{\left(B_{s} N_{0}+P_{l}^{(H)} G_{l}^{(L)}\right)\left(1+\gamma_{l}^{(L)}\right)}-\psi \\
B_{s=1}^{S} \log _{2}\left(1+\gamma_{l}^{(L)}\right) \\
\frac{\Phi_{\min }^{(L)}}{d \psi}=\sum_{l=1}^{S}\left(P_{l}^{(L)}+P_{l}^{(H)}\right)-P_{t} . \\
\log _{2}\left(1+\gamma_{l}^{(H)}\right)
\end{gathered}
$$

To simplify the solution, we assume the total transmit power is divided equally to each data stream, which is then optimally allocated to the strong and weak users within the stream. We denote this the equal power allocation (EPA) scheme. With this assumption and setting each of these equations to zero and solving (24) for $P_{l}^{(L)}$ we get

$$
P_{l}^{(L)}=\frac{P_{t}}{S}-P_{l}^{(H)}=P_{T S}-P_{l}^{(H)} .
$$

Finally, substituting (25) into (23), the sub-optimal power for the strong user is found to be

$$
P_{l}^{(H)}=\left[\frac{\sqrt{B_{s} N_{0} \beta}-B_{s} N_{0} \sqrt{\Gamma_{H}}\left(G_{l}^{(H)}+G_{l}^{(L)}\right)}{2 G_{l}^{(H)} G_{l}^{(L)} \sqrt{\Gamma_{H}}}\right]^{+}
$$

where $\quad \beta=4 G_{l}^{(H)} G_{l}^{(L)} \Gamma_{L}\left(B_{s} N_{0}+G_{l}^{(L)} P_{T S}\right)+$ $B_{s} N_{0} \Gamma_{H}\left(G_{l}^{(H)}-G_{l}^{(L)}\right)^{2}$. by

By substituting (26) in (25), the expression of $P_{l}^{(L)}$ is given

$$
\begin{array}{r}
P_{l}^{(L)}=\left[\frac{2 G_{l}^{(H)} G_{l}^{(L)} \sqrt{\Gamma_{H}} P_{T S}-\sqrt{B_{s} N_{0} \beta}}{2 G_{l}^{(H)} G_{l}^{(L)} \sqrt{\Gamma_{H}}}-\right. \\
\left.\frac{B_{s} N_{0} \sqrt{\Gamma_{H}}\left(G_{l}^{(H)}+G_{l}^{(L)}\right)}{2 G_{l}^{(H)} G_{l}^{(L)} \sqrt{\Gamma_{H}}}\right]^{+}
\end{array}
$$

where $\Gamma_{H}=2^{\frac{1}{\Phi_{m i n}^{(H)}}}$ and $\Gamma_{L}=2^{\frac{1}{\Phi_{m i n}^{(L)}}}$.

These two equations represent the proposed EPA scheme which will be used to allocate the user power when only two 
users are multiplexed per each data stream, while the rest are aligned to the interference subspace.

\section{Extension to Multiuser Power Allocation}

To apply the proposed EPA solutions in (26) and (27), the concept of hierarchical pairing (HP) which was presented in [14] will be used to form the proposed hierarchical pairing based power allocation scheme (HPPA). According to HP, the users are grouped into $Z$ pairs, and the pairs are arranged in an ascending order according to their channel powers. By dividing the user-pairs into two groups and combining their channel powers, the power allocation solutions (26) and (27) can be modified to obtain the power allocated to each group. Following the principles of NOMA, the stronger half of the users will form one group, and the weaker half will form the other. Hence, the terms $G_{l}^{(H)}$ and $G_{l}^{(L)}$ in (26) and (27) will become the sum of all channel powers in the stronger and weaker group respectively. Once the powers of the two groups are obtained, the same procedure is repeated to each group as a second stage; i.e., two subgroups are formed in each group of the previous stage. This multiple stage approach is repeated until the subgroups become pairs, and the solution (26) and (27) can be used directly. To perform SIC detection, the weakest user in the first pair (the weakest user-pair) will not perform SIC while the better user in this pair will perform SIC only to its partner in this pair. At the last pair (the strongest user-pair), on the other hand, the weaker user of the $Z$-th pair will perform SIC to all of the previous pairs, while the strongest user at this pair will perform SIC to all of the previous pairs and its partner as well. To sum up, the achievable rate by the weak user in the $z$-th pair is given by

$$
R_{z}^{(L)}=B_{s} \sum_{l=1}^{S} \log _{2}\left(1+\frac{P_{z, l}^{(L)} G_{z, l}^{(L)}}{B_{s} N_{0}+I_{z}+P_{z, l}^{(H)} G_{z, l}^{(L)}}\right)
$$

while that achieved by the better user in this pair is given by

$$
R_{z}^{(H)}=B_{s} \sum_{l=1}^{S} \log _{2}\left(1+\frac{P_{z, l}^{(H)} G_{z, l}^{(H)}}{I_{z}+B_{s} N_{0}}\right)
$$

where $I_{z}$ represent the interference from other pairs, and it is given by

$$
I_{z}=\left\{\begin{array}{c}
z=Z \\
\sum_{c=z+1}^{Z}\left(P_{c, l}^{(L)} G_{z, l}\right)+\left(P_{c, l}^{(H)} G_{z, l}\right) \quad 1 \leq z \leq(Z-1) .
\end{array}\right.
$$

By applying HPPA, the expression of the strong user is given by

$$
\begin{gathered}
P_{l}^{(H)}=\left[-\frac{B_{s} N_{0} \sqrt{\Gamma_{H}}\left(G_{l}^{(H)}+G_{l}^{(L)}\right)-2 I_{z} G_{l}^{(H)} G_{l}^{(L)} \sqrt{\Gamma_{H}}}{2 G_{l}^{(H)} G_{l}^{(L)} \sqrt{\Gamma_{H}}}+\right. \\
\left.\frac{\sqrt{\Omega+\left(B_{s} N_{0}\right)^{2} \Gamma_{H}\left(G_{l}^{(H)}-G_{l}^{(L)}\right)^{2}}}{2 G_{l}^{(H)} G_{l}^{(L)} \sqrt{\Gamma_{H}}}\right]^{+}
\end{gathered}
$$

and that of the weak user is given by

$$
\begin{gathered}
P_{l}^{(L)=}\left[\frac{B_{s} N_{0} \sqrt{\Gamma_{H}}\left(G_{l}^{(H)}+G_{l}^{(L)}\right)+2\left(P_{T S}+I_{z}\right) G_{l}^{(H)} G_{l}^{(L)} \sqrt{\Gamma_{H}}}{2 G_{l}^{(H)} G_{l}^{(L)} \sqrt{\Gamma_{H}}}-\right. \\
\left.\frac{\sqrt{\Omega+\left(B_{s} N_{0}\right)^{2} \Gamma_{H}\left(G_{l}^{(H)}-G_{l}^{(L)}\right)^{2}}}{2 G_{l}^{(H)} G_{l}^{(L)} \sqrt{\Gamma_{H}}}\right]^{+}
\end{gathered}
$$

where $\Omega=4 G_{l}^{(H)} G_{l}^{(L)} \Gamma_{L}\left(B_{s} N_{0}+G_{l}^{(H)} I_{z}\right)\left(B_{s} N_{0}+G_{l}^{(L)}\left(I_{z}+P_{T S}\right)\right)$.

\section{E. Subgradient based Power Allocation}

Another approach can be obtained by setting (22) to zero and solving with respect to $P_{l}^{(L)}$

$$
\begin{gathered}
\frac{d F}{d P_{l}^{(L)}}=\frac{\mu B_{s} G_{l}^{(L)}}{\Phi_{\min }^{(L)}\left(B_{s} N_{0}+P_{l}^{(H)} G_{l}^{(L)}\right)\left(1+\frac{P_{l}^{(L)} G_{l}^{(L)}}{B_{s} N_{0}+P_{l}^{(H)} G_{l}^{(L)}}\right)}+ \\
\frac{B_{s} \lambda_{l}^{(L)}}{\left(B_{s} N_{0}+P_{l}^{(H)} G_{l}^{(L)}\right)\left(1+\frac{P_{l}^{(L)} G_{l}^{(L)}}{B_{s} N_{0}+P_{l}^{(H)} G_{l}^{(L)}}\right)}-\psi=0 \\
P_{l}^{(L)}=\left[\frac{\mu+\Phi_{\min }^{(L)}}{\psi \Phi_{\min }^{(L)}}-\frac{B_{s} N_{0}+P_{l}^{(H)} G_{l}^{(L)}}{B_{s} G_{l}^{(L)}}\right]^{+}
\end{gathered}
$$

Similarly, the expression of $P_{l}^{(H)}$ is obtained to be

$$
P_{l}^{(H)}=\left[\frac{\mu+\Phi_{\min }^{(H)}}{\psi \Phi_{\min }^{(H)}}-\frac{N_{0}}{G_{l}^{(H)}}\right]^{+} .
$$

From these two expressions, the power allocation can be concluded to be applied for multiple number of users as follows, the power allocated to all the users except the strongest one is given by

$$
P_{k, l}=\left[\frac{\left(\mu_{k}+\Phi_{\min }^{(k)}\right)}{\psi \Phi_{\min }^{(k)}}-\frac{B_{s} N_{0}+\sum_{m=k+1}^{K} P_{m, l} G_{l}^{(k)}}{B_{s} G_{l}^{(k)}}\right]^{+} .
$$

On the other hand, the expression for the strongest user is set to be

$$
P_{K, l}=\left[\frac{\mu_{K}+\Phi_{\min }^{(K)}}{\psi \Phi_{\min }^{(K)}}-\frac{N_{0}}{G_{l}^{(K)}}\right]^{+} .
$$

Following the steps of Algorithm 1, the optimal power allocation can be obtained. At each step of this algorithm, the Lagrangian multipliers will be updated using

$$
\begin{gathered}
\lambda_{k}^{(i+1)}=\left[\lambda_{k}^{(i)}-\Theta^{(i)}\left(P_{t}-\sum_{k=1}^{K} \sum_{l=1}^{S} P_{k, l}\right)\right]^{+} \\
\mu_{k}^{(i+1)}=\left[\mu_{k}^{(i)}-\Upsilon^{(i)}\left(\sum _ { k = 1 } ^ { K - 1 } \left(\Phi_{k} B_{s} \sum_{l=1}^{S} \log _{2}\left(1+\gamma_{k, l}\right)\right.\right.\right. \\
\left.\left.\left.-\Phi_{K} B_{s} \sum_{l=1}^{S} \log _{2}\left(1+\gamma_{K, l}\right)\right)\right)\right]^{+}
\end{gathered}
$$


Table I

SIMULATION PARAMETERS [16]

\begin{tabular}{|c|c|}
\hline Parameter Name & Value \\
\hline Cell diameter $(D)$ & $300 \mathrm{~m}$ \\
\hline Path loss exponent $(v)$ & 3.76 \\
\hline Noise power density $\left(N_{0}\right)$ & $-174 \mathrm{dBm} / \mathrm{Hz}$ \\
\hline Bandwidth per RB $\left(B_{s}\right)$ & $200 \mathrm{kHz}$ \\
\hline No. of users $(K)$ & 4 \\
\hline No. of transmitting antennas $(M)$ & 8 \\
\hline No. of receiving antennas $(N)$ & 2 \\
\hline Shadowing standard deviation & $8 \mathrm{~dB}$ \\
\hline$P L_{0}$ at $2 \mathrm{GHz}$ band & $15.3+37.6 \log _{10}\left(d_{0}\right)$ \\
\hline$\Phi_{\min }^{(H)}$ & $1 \mathrm{Mbps}$ \\
\hline$\Phi_{\min }^{(L)}$ & $0.5 \mathrm{Mbps}$ \\
\hline
\end{tabular}

where $\Theta^{(i)}$ and $\Upsilon^{(i)}$ are small step sizes to be updated at each iteration and chosen to be $0.1 / \sqrt{i}$ [15].

The solution to the problem in (15)-(19) is summarized in Algorithm 1.

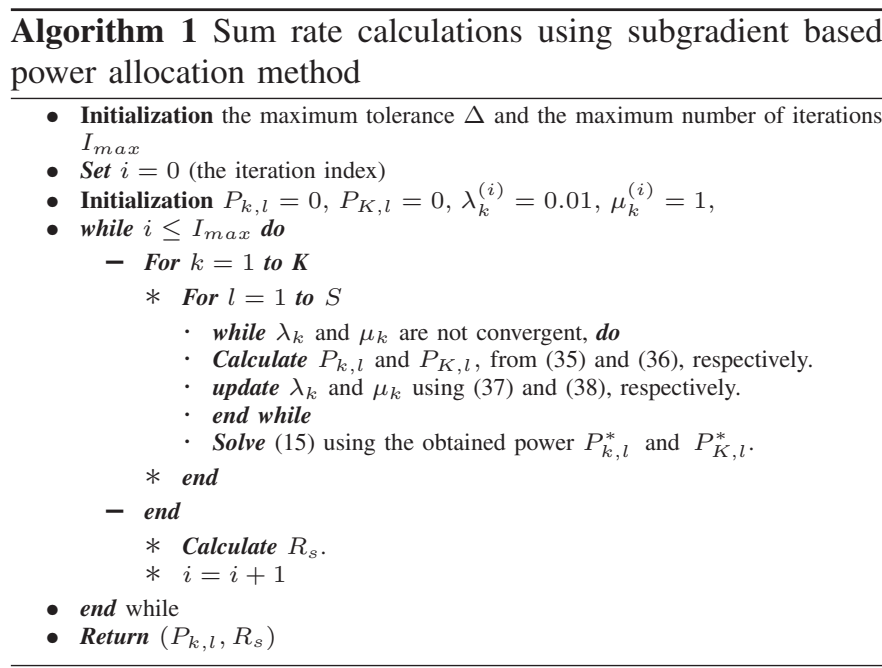

\section{SIMULATION RESULTS}

A downlink MIMO-NOMA system is simulated with $K$ users distributed uniformly in a circular area of diameter $300 \mathrm{~m}$. Other simulation parameters are listed in Table I. The proposed EPA, HPPA, and the subgradient methods will be compared against the numerically obtained optimal NOMA and FTPA based NOMA [6]. In addition, channel estimation is assumed to be perfectly applied and the CSI is assumed to be perfectly known at the BS.

The simulated scenario considers applying IA to the twouser per stream case and then comparing it to that of multiuser per stream case. Where for each user pair, SVD-IA will align all the other pairs into an interference subspace and NOMA will be applied within the data stream. Fig. 1 and 2 show the improvement of the two-user per stream case with IA as compared to the multiuser per stream case. This is a clear proof that IA could further enhance the sum rate for NOMA system and also simplifies the complexity of SIC as with IA each user apply SIC to its partner only while all other users were aligned by IA. In addition, these figures show that DPC based schemes offers better sum rate than their SVD based counterparts. For example, the optimal scheme of multiplexing two users per stream with the DPC scheme in Fig. 2 offers better sum rate than its SVD based counterpart depicted in Fig. 1. Moreover, the optimal scheme for multiplexing all the users per a data stream with DPC which is illustrated in Fig. 2 outperforms that with SVD which is illustrated in Fig. 1. This is also the case for the subgradient and the FTPA schemes.

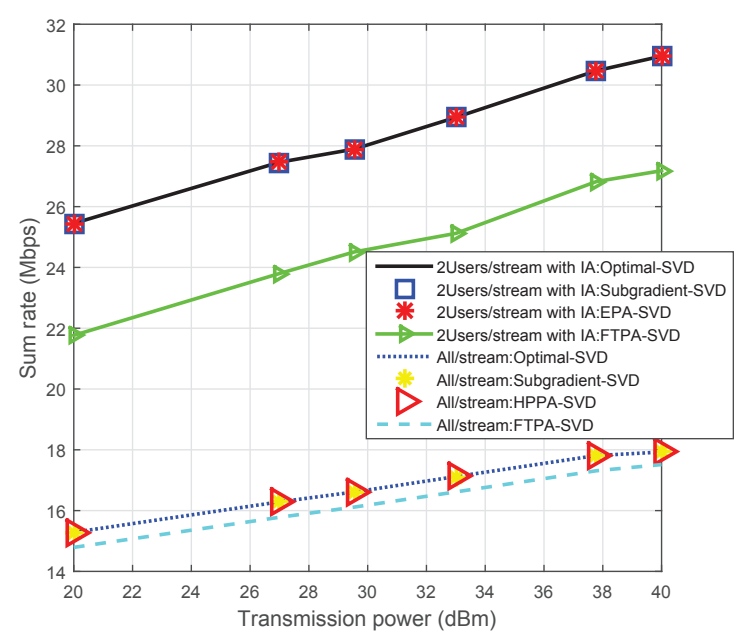

Fig. 1. Sum rate versus various transmission power levels with SVD-IA and SVD precoding.

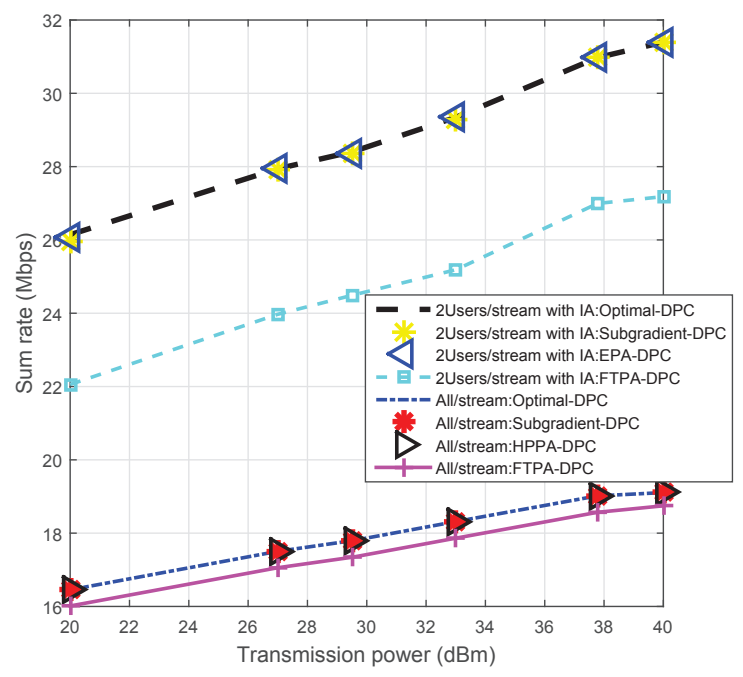

Fig. 2. Sum rate versus various transmission power levels with SVD-IA and DPC precoding.

Next, the comparison is made in terms of the sum rate against the increasing number of users. Fig. 3 and 4 show the increasing sum rate in proportion to the number of users, which is due to the multiuser diversity gain acquired by using NOMA. These figures also confirm that IA can improve the achievable sum rate and reduce the SIC complexity for a large number of users. In addition, Fig. 4 shows that using DPC precoding based schemes outperforms the same schemes based on SVD precoding which are shown in Fig. 3. For instance, 


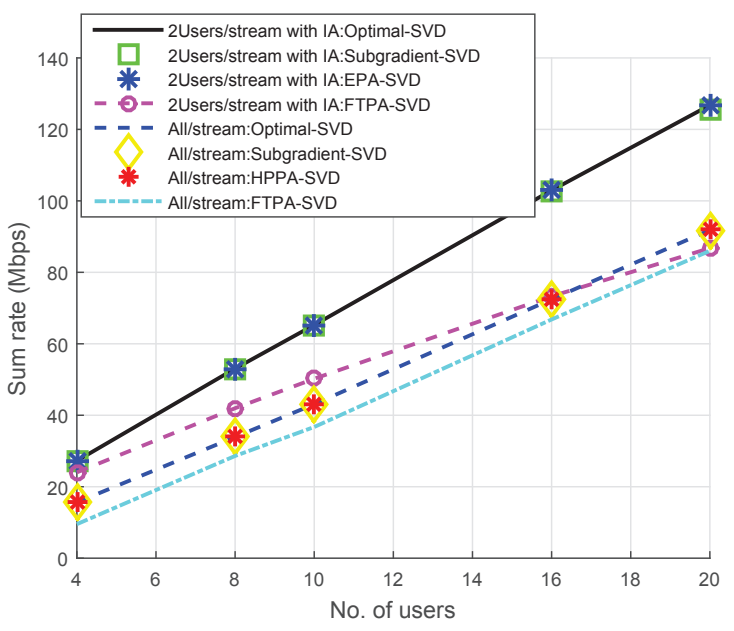

Fig. 3. Sum rate versus increasing number of users with SVD-IA and SVD precoding with $P_{t}=30 \mathrm{dBm}$.

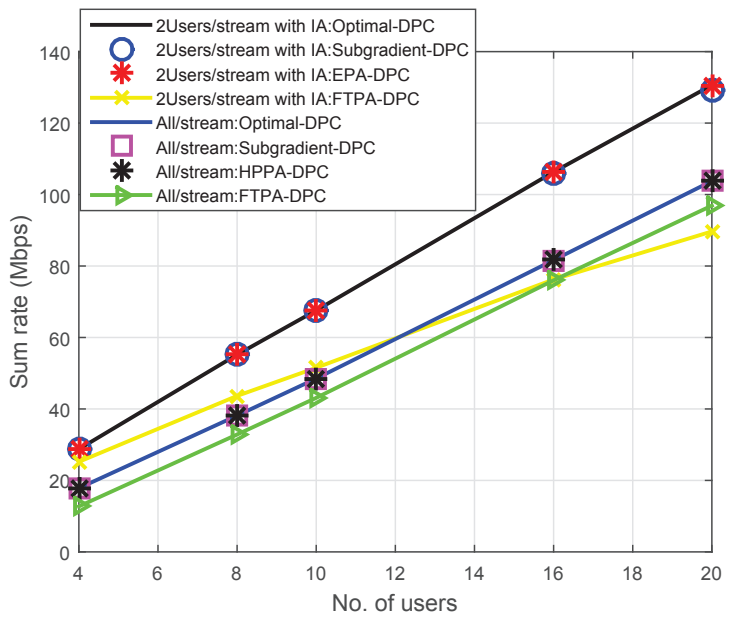

Fig. 4. Sum rate versus increasing number of users with SVD-IA and DPC precoding with $P_{t}=30 \mathrm{dBm}$.

for a two users per stream case, the proposed subgradient-DPC technique displayed in Fig. 4 shows better performance than the proposed subgradient-SVD technique illustrated in Fig. 3. Moreover, for the all-users per stream case, the optimal-DPC scheme in Fig. 4 offers better sum rate than the optimal SVD scheme depicted in Fig. 3, this is also the case for all of the compared schemes as can be noticed by examining Fig. 3 and 4 , which confirms our previous findings.

It is worth mentioning that the proposed schemes offer the advantage of simplicity as compared to the optimal approach. In particular, the proposed HPPA has the lowest complexity of about $(K-1) * M$ times of (31) and (32) computations while the subgradient one has a complexity of $\mathcal{O}\left(\frac{M K}{\left(1 / \Delta^{2}\right)}\right)$ times of water-filling computations in (35) and (36).

\section{CONCLUSION}

This paper presents new resource allocation schemes for MIMO-NOMA system with IA. A sum rate maximization problem is formulated under the total transmit power constraint and the minimum rate constraint. First, EPA is obtained as a two-user based suboptimal solution to allocate the power per each user. Then the solution is expanded to a multiuser using hierarchical pairing approach to propose HPPA. A subgradient based approach is also proposed to allocate the power iteratively to each user. Simulations are used to compare the proposed schemes against the numerically obtained optimal scheme along with the FTPA scheme from the literature. From the obtained results, it is clear that all of the proposed schemes (e.g., EPA, HPPA and the subgradient method) outperform FTPA and also show close performance to the optimal scheme. Moreover, simulations show that applying IA with NOMA offers better sum rate and requires simpler SIC. In particular, all of the proposed schemes as well as the optimal one offered better sum rate performance if the precoding is applied based on DPC rather than SVD.

\section{REFERENCES}

[1] H. Q. Ngo, E. G. Larsson, and T. L. Marzetta, "Energy and spectral efficiency of very large multiuser mimo systems," IEEE Transactions on Communications, vol. 61, no. 4, pp. 1436-1449, April 2013.

[2] F.-L. Luo and C. Zhang, Signal Processing for 5G: Algorithms and Implementations. John Wiley \& Sons, 2016.

[3] Q. Sun, S. Han, Z. Xu, S. Wang, I. Chih-Lin, and Z. Pan, "Sum rate optimization for mimo non-orthogonal multiple access systems," in Wireless Communications and Networking Conference (WCNC), 2015 IEEE, March 2015, pp. 747-752.

[4] Z. Ding, R. Schober, and H. V. Poor, "A general mimo framework for noma downlink and uplink transmission based on signal alignment," IEEE Transactions on Wireless Communications, vol. 15, no. 6, pp. 4438-4454, June 2016.

[5] Y. Sun, D. W. K. Ng, Z. Ding, and R. Schober, "Optimal joint power and subcarrier allocation for mc-noma systems," arXiv preprint arXiv:1603.08132, 2016.

[6] J. Kim, J. Koh, J. Kang, K. Lee, and J. Kang, "Design of user clustering and precoding for downlink non-orthogonal multiple access (noma)," in Military Communications Conference, MILCOM 2015 - 2015 IEEE, Oct 2015, pp. 1170-1175.

[7] Y. Liu, G. Pan, H. Zhang, and M. Song, "On the capacity comparison between mimo-noma and mimo-oma," IEEE Access, vol. 4, pp. 21232129, 2016.

[8] Q. Sun, S. Han, C.-L. I, and Z. Pan, "Energy efficiency optimization for fading mimo non-orthogonal multiple access systems," in Communications (ICC), 2015 IEEE International Conference on, June 2015, pp. 2668-2673.

[9] A. Li, A. Benjebbour, and A. Harada, "Performance evaluation of nonorthogonal multiple access combined with opportunistic beamforming," in Vehicular Technology Conference (VTC Spring), 2014 IEEE 79th, May 2014, pp. 1-5.

[10] Q. Sun, S. Han, C. L. I, and Z. Pan, "On the ergodic capacity of mimo noma systems," IEEE Wireless Communications Letters, vol. 4, no. 4, pp. 405-408, Aug 2015.

[11] K. Ronasi, B. Niu, V. W. Wong, S. Gopalakrishnan, and R. Schober, "Throughput-efficient scheduling and interference alignment for mimo wireless systems," IEEE Transactions on Wireless Communications, vol. 13, no. 4, pp. 1779-1789, 2014.

[12] J. Tang, D. K. C. So, E. Alsusa, K. A. Hamdi, and A. Shojaeifard, "Energy efficiency optimization with interference alignment in multicell mimo interfering broadcast channels," IEEE Transactions on Communications, vol. 63, no. 7, pp. 2486-2499, July 2015.

[13] S. Boyd and L. Vandenberghe, Convex optimization. Cambridge university press, 2004.

[14] Z. Q. Al-Abbasi and D. K. C. So, "User-pairing based non-orthogonal multiple access (noma) system," in 2016 IEEE 83rd Vehicular Technology Conference (VTC Spring), May 2016, pp. 1-5.

[15] S. Boyd, L. Xiao, and A. Mutapcic, "Subgradient methods," lecture notes of EE392o, Stanford University, Autumn Quarter, vol. 2004, pp. 2004-2005, 2003.

[16] G. Auer, V. Giannini, C. Desset, I. Godor, P. Skillermark, M. Olsson, M. Imran, D. Sabella, M. Gonzalez, O. Blume, and A. Fehske, "How much energy is needed to run a wireless network?" Wireless Communications, IEEE, vol. 18, no. 5, pp. 40-49, October 2011. 